\title{
Current Status and Future Perspectives of the COBRA Experiment
}

\author{
J. Ebert, ${ }^{1}$ M. Fritts, ${ }^{2}$ C. Gößling, ${ }^{3}$ T. Göpfert, ${ }^{2}$ D. Gehre, ${ }^{2}$ C. Hagner, ${ }^{1}$ N. Heidrich, \\ T. Köttig, ${ }^{3}$ T. Neddermann, ${ }^{3}$ C. Oldorf, ${ }^{1}$ T. Quante, ${ }^{3}$ S. Rajek, ${ }^{3}$ O. Reinecke, ${ }^{2}$ O. Schulz, \\ J. Tebrügge, ${ }^{3}$ J. Timm, ${ }^{1}$ B. Wonsak, ${ }^{1}$ and K. Zuber ${ }^{2}$ \\ ${ }^{1}$ Institut für Experimentalphysik, Universität Hamburg, Luruper Chaussee 149, 22761 Hamburg, Germany \\ ${ }^{2}$ Institut für Kern- und Teilchenphysik, Technische Universität Dresden, Zellescher Weg 19, 01068 Dresden, Germany \\ ${ }^{3}$ Lehrstuhl für Experimentelle Physik IV, Technische Universität Dortmund, Otto-Hahn-Straße 4, 44221 Dortmund, Germany \\ ${ }^{4}$ Max-Planck-Institut für Physik, Werner-Heisenberg-Institut, Foehringer Ring 6, 80805 München, Germany \\ Correspondence should be addressed to M. Fritts; matthew_christopher.fritts@tu-dresden.de
}

Received 5 July 2013; Accepted 30 August 2013

Academic Editor: Vincenzo Flaminio

Copyright (C) 2013 J. Ebert et al. This is an open access article distributed under the Creative Commons Attribution License, which permits unrestricted use, distribution, and reproduction in any medium, provided the original work is properly cited.

\begin{abstract}
The aim of the COBRA experiment is to prove the existence of neutrinoless double-beta-decay ( $0 \nu \beta \beta$-decay) and to measure its half-life. For this purpose a detector array made of cadmium-zinc-telluride (CdZnTe) semiconductor detectors is operated at the Gran Sasso Underground Laboratory (LNGS) in Italy. This setup is used to investigate the experimental issues of operating CdZnTe detectors in low-background mode and to identify potential background components, whilst additional studies are proceeding in surface laboratories. The experiment currently consists of monolithic, calorimetric detectors of coplanar grid design (CPG detectors). These detectors are $1 \times 1 \times 1 \mathrm{~cm}^{3}$ and are arranged in $4 \times 4$ detector layers. Ultimately four layers will be installed by the end of 2013, of which two are currently operating. To date $82.3 \mathrm{~kg}$.days of data have been collected. In the region of interest for ${ }^{116} \mathrm{Cd}$ around $2.8 \mathrm{MeV}$, the median energy resolution is $1.5 \% \mathrm{FWHM}$, and a background level near 1 counts $/ \mathrm{keV} / \mathrm{kg} / \mathrm{y}$ has been reached. This paper gives an overview of the current status of the experiment and future perspectives.
\end{abstract}

\section{Introduction}

Among the outstanding issues of modern particle physics are measuring the absolute mass scale of the neutrino and the determination of whether neutrinos are Majorana or Dirac particles. This motivates searching for $0 \nu \beta \beta$-decay, because its existence would be an unambiguous sign for the Majorana character of the neutrino and would be a probe of the absolute mass. Additionally, as a lepton number violating process, $0 \nu \beta \beta$-decay is a probe of physics beyond the standard model [1]. Searches for $0 \nu \beta \beta$-decay require a source of double-betadecay isotopes and a low-background detection system. The Cadmium Zinc Telluride 0-Neutrino Double-Beta Research Apparatus (COBRA) experiment, proposed in [2], uses CdZnTe crystals as both source and detector material. Within this material, nine isotopes are double-beta-decay candidates (see Table 1), the most promising being ${ }^{130} \mathrm{Te}$ and ${ }^{116} \mathrm{Cd}$. The main virtue of ${ }^{130} \mathrm{Te}$ is its very high natural abundance of $33.8 \%$, whereas ${ }^{116} \mathrm{Cd}$ is superior due to its higher $\mathrm{Q}$-value of $2814 \mathrm{keV}$, which lies above the ${ }^{208} \mathrm{Tl}$ peak at $2615 \mathrm{keV}$, the highest energy gamma line of significant intensity from natural radioactivity. This advantage is quite crucial: not only does a higher Q-value increase the phase space-and thus the probability-for the sought-after decay, but it also reduces the gamma background from natural radioactivity by more than one order of magnitude with respect to isotopes with $Q$-values below the ${ }^{208} \mathrm{Tl}$ peak. In contrast to other semiconductor or bolometric detectors, CdZnTe crystals have the convenience that they can be operated at room temperature, still providing an excellent energy resolution of a few percent. Good resolution is important to distinguish signal from existing backgrounds, including the unavoidable intrinsic background originating from the conventional double-betadecay. CdZnTe detectors are commercially available, making 
TABLE 1: List of $0 v \beta \beta$-decay isotopes in CdZnTe with their Q-values and natural abundance.

\begin{tabular}{lccc}
\hline Isotope & nat. ab. $(\%)$ & Q-value $(\mathrm{keV})$ & Decay mode \\
\hline${ }^{70} \mathrm{Zn}$ & 0.62 & 1001 & $\beta^{-} \beta^{-}$ \\
${ }^{114} \mathrm{Cd}$ & 28.7 & 534 & $\beta^{-} \beta^{-}$ \\
${ }^{116} \mathrm{Cd}$ & 7.5 & 2814 & $\beta^{-} \beta^{-}$ \\
${ }^{128} \mathrm{Te}$ & 31.7 & 868 & $\beta^{-} \beta^{-}$ \\
${ }^{130} \mathrm{Te}$ & 33.8 & 2527 & $\beta^{-} \beta^{-}$ \\
${ }^{64} \mathrm{Zn}$ & 48.6 & 1096 & $\beta^{+} / \mathrm{EC}$ \\
${ }^{106} \mathrm{Cd}$ & 1.21 & 2771 & $\beta^{+} \beta^{+}$ \\
${ }^{108} \mathrm{Cd}$ & 0.9 & 231 & $\mathrm{EC} / \mathrm{EC}$ \\
${ }^{120} \mathrm{Te}$ & 0.1 & 1722 & $\beta^{+} / \mathrm{EC}$ \\
\hline
\end{tabular}

them comparatively inexpensive. Furthermore, they have proven to be very radiopure, which is crucial for avoiding intrinsic background.

In this paper the current status of the COBRA test setup as well as recent results from the data collected is presented. In addition, prospects for a large-scale setup and a sensitivity projection are given.

\section{Experimental Setup}

The COBRA R\&D low-background test setup is situated in the Italian underground laboratory LNGS. An overburden of 1400 meters of rock coverage ( 3600 m.w.e.) reduces the cosmic ray flux by about six orders of magnitude.

The current setup is able to handle a total of sixty-four $1 \mathrm{~cm}^{3} \mathrm{CdZnTe}$ crystals. It consists of four layers, each capable of holding $4 \times 4$ detector crystals in a Delrin support. To reduce the flux of external gammas, the inner detector chamber is shielded by $5 \mathrm{~cm}$ ultra-radiopure electrolytic copper and $20 \mathrm{~cm}$ low-radioactivity lead. Supply of bias voltages and signal readout occurs via low-diameter coaxial cables and copper traces on Kapton foils which are fed through the shielding (Figure 1(a)). Around this an EMI shielding made of galvanized steel sheets is installed, housing also the preamplifiers of the readout chain (Figure 1(b)). A $7 \mathrm{~cm}$ outer layer of boron-loaded polyethylene shields against neutrons. To prevent contamination with radon, the setup is constantly flushed with nitrogen gas. Two layers (32 detectors) have been in operation since April 2012.

The data acquisition system records complete pulse shapes for each event using fast analog-digital converters. This system is designed to optimize event reconstruction and to allow for event discrimination through pulse shape analysis [3]. Frequent calibrations with gamma radiation sources allow for the calculation of the detectors' amplification and resolution properties, which vary slowly over constant running conditions within a few percent. The setup accumulates data at a rate of approximately $2.5 \mathrm{~kg} \cdot$ days per layer per month.

Recently a pulser was installed to send fixed-amplitude, simultaneous signals to the preamplifiers (two per detector). This provides an additional test of the stability of the system components. Moreover, these signals provide for synchronization of the clocks in the eight ADC modules (one ADC module for every four detectors) which record the signals. This allows for event coincidence studies over the full detector array with a time resolution smaller than $0.5 \mathrm{~ms}$. In June 2013, the third layer of detectors was installed as well as a cooling system, which dispenses the heat of the preamplifiers and should slightly improve the energy resolution [4]. The fourth layer is expected to be installed by the end of 2013 .

\section{CPG Capabilities}

The current setup at LNGS is equipped with coplanar grid (CPG) detectors. COBRA is also exploring the use of pixelized detectors, which have the potential to further reduce the background using topological information. These efforts are not considered in this paper but are discussed in [5].

The CPG technology was developed as a method to compensate for the poor transport properties of holes in CdZnTe [6]. With this readout approach, energy resolutions better than 2\% FWHM at $662 \mathrm{keV}$ can be achieved with commercially available detectors (see Figure 2). One side of a CPG detector is a uniform cathode, while two anode grids are patterned on the opposite side in the form of two interlocking combs (Figure 3). These anodes are held at slightly different potentials and are read out separately. The amplitudes of the two pulses form the basis of event reconstruction.

In addition to energy information, the amplitudes of the two anode signals provide depth information [7]. The interaction depth is defined as the distance from the anode surface of the detector expressed as a fraction of the detector length. Figure 4 shows the distribution of background events in energy and interaction depth. At very low depths, distortions in energy reconstruction occur as a consequence of the CPG design. At the cathode surface $($ depth $=1)$ contamination from alpha emitters produces high energy event populations. Both the low-depth distortions and the high-depth surface events can be efficiently removed using the interaction depth information.

The ability to identify events at the lateral surfaces of the detector is also useful for background reduction. Tests with alpha sources have shown that interactions very near the lateral surfaces of the detector produce signature distortions in the pulses as shown in Figure 5. These distortions can be characterized by pulse shape analysis. Simulations suggest that about $80 \%$ of surface events are identifiable in this way.

Another use for pulse shape analysis is the identification and rejection of events caused by electronic disturbances, some of which can mimic real events in terms of energy and depth reconstruction. Pulse shape analysis can also be used for the identification of events in which energy is deposited at two or more distinct depths within a detector (multisite events). This is useful for reducing potential gamma backgrounds.

\section{Current Status}

To date COBRA has collected $82.3 \mathrm{~kg}$.days of calibrated lowbackground exposure. With this data a thorough characterization of the background in the LNGS setup is possible. Figure 6 depicts the background rate in standardized 


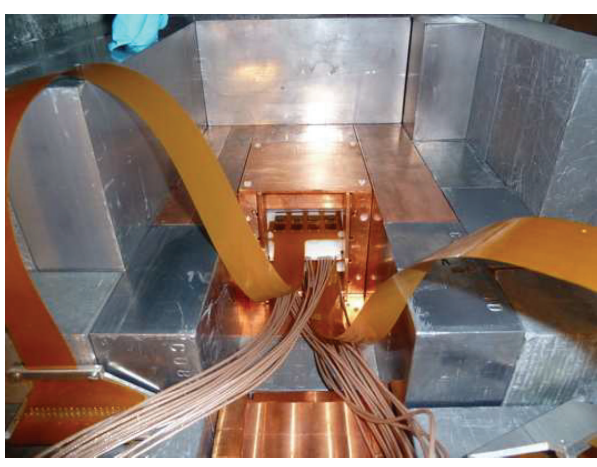

(a)

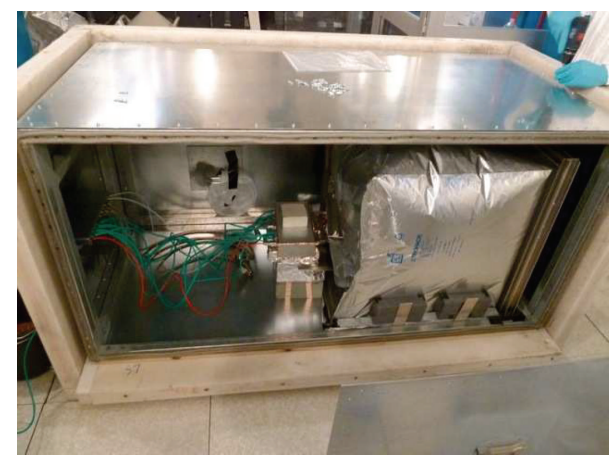

(b)

Figure 1: (a) Picture of the inner part of the setup showing the copper nest and the cable feedthrough surrounded by the lead and copper castle. (b) Picture of the whole setup with open EMI and neutron shielding showing the radon-tight foil containing the lead bunker.

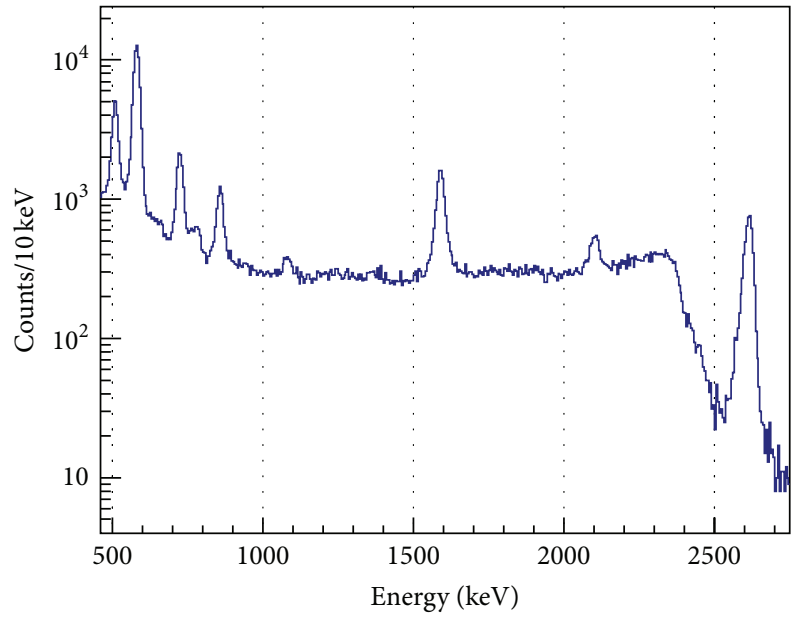

(a)

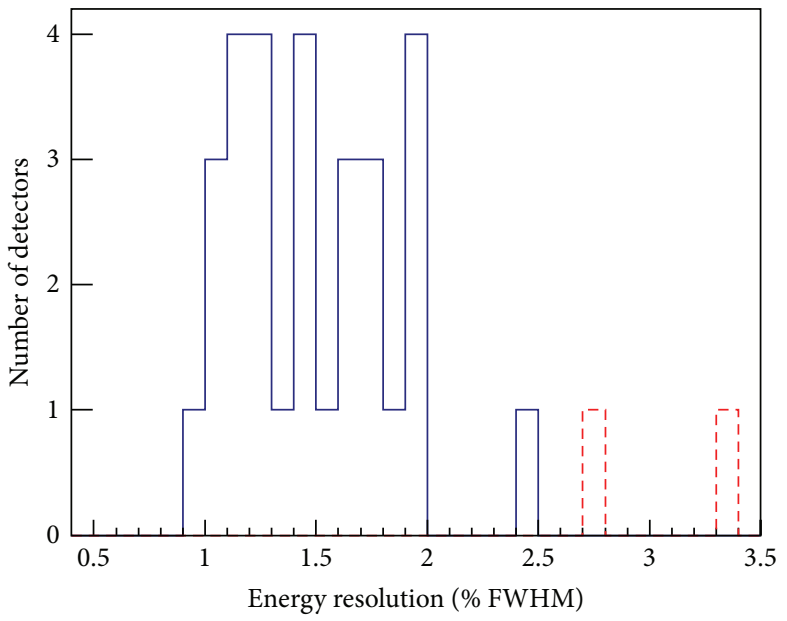

(b)

Figure 2: (a) Spectrum for a typical COBRA detector from an in situ ${ }^{228} \mathrm{Th}$ calibration. Calibration lines from ${ }^{22} \mathrm{Na}$ and ${ }^{228} \mathrm{Th}$ calibrations are used to characterize the resolution as a function of energy. (b) Resolutions of the 32 currently operating detectors extrapolated to $2814 \mathrm{keV}$. The worst two detectors are from an older batch and are not representative of detectors that will be deployed in the future. The median value is $1.5 \%$ FWHM.

units and shows the effectiveness of discriminating surface background. At the region of interest near $2.8 \mathrm{MeV}$, the background rate is reduced by about an order of magnitude by removing events at the cathode and lateral surfaces, down to approximately 1 counts $/ \mathrm{keV} / \mathrm{kg} / \mathrm{y}$. Throughout this paper background rates are given per unit mass of detector material (CdZnTe). The relative efficiencies for $0 \nu \beta \beta$-decay signals of these cuts are estimated at $95 \%$ for the cathode cut and $82 \%$ for the lateral surface cut. Two alpha peaks are evident in the cathode surface events, but no peaks are seen in the lateral surface events. This is expected because the cathode side is covered by only about $200 \mathrm{~nm}$ of metal, whereas the lateral surfaces are encapsulated in transparent paint some $10 \mathrm{~s}$ of $\mu \mathrm{m}$ thick. Thus, the energy of alpha radiation from the lateral surfaces is attenuated. Nonuniformity in the paint thickness will generally lead to a continuous spectrum.

Figure 4 shows that even after removing high- and lowdepth background, the interaction depth distribution is not uniform. The band of more intense background at an interaction depth between 0.75 and 0.95 may be due to alpha contamination on the detector support structure. Depending on the analysis technique used, the depth dependence of the background can be exploited to reduce the background for the $0 \nu \beta \beta$-decay search. The effective background rate based on an optimized sensitivity calculation at $2.8 \mathrm{MeV}$ can be reduced to approximately 0.4 counts $/ \mathrm{keV} / \mathrm{kg} / \mathrm{y}$. The signal efficiency for the optimized sensitivity is approximately $95 \%$ relative to the nonoptimized case.

Under current experimental conditions, the full array of 64 detectors should achieve a sensitivity to the ${ }^{116} \mathrm{Cd} 0 \nu \beta \beta$ decay half-life of $2.3 \times 10^{21} \mathrm{y}$ after 2 years of live time. However, ideas currently under development for improving the performance of the experiment may increase this estimate. At present the spectrum from the conventional double-betadecay of ${ }^{116} \mathrm{Cd}$ is just visible in certain energy ranges after all background-reducing cuts are applied. 


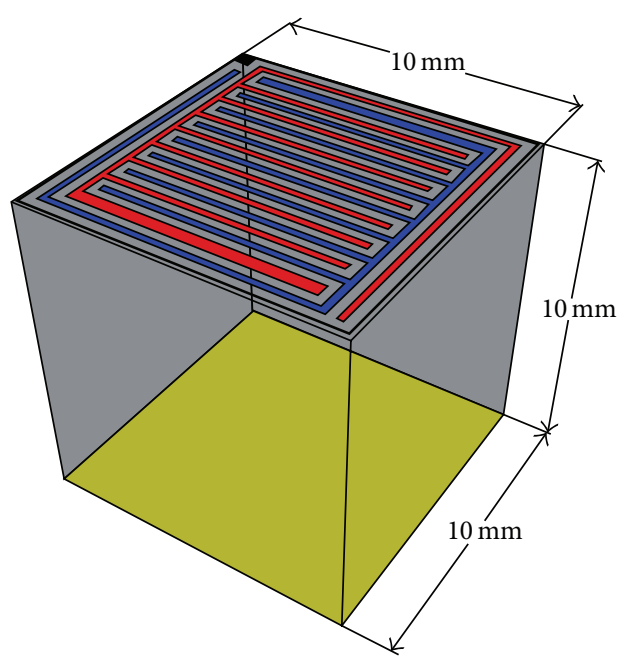

(a)

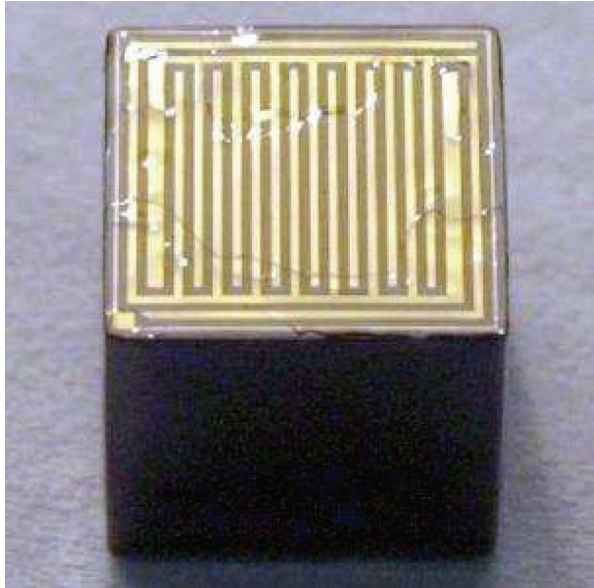

(b)

FIGURE 3: (a) Diagram of CPG detector indicating the anode grids (blue and red) and the cathode (yellow). (b) Photograph of a COBRA detector.

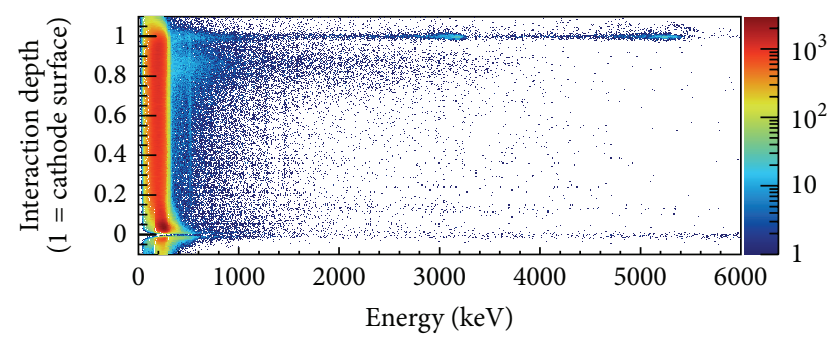

FIGURE 4: Distribution of events in interaction depth and energy from $82.3 \mathrm{~kg} \cdot$ days of low-background data. Two high energy regions on the cathode surface are identified as alpha contaminants. Three gamma lines (vertical) are also visible. The region below $400 \mathrm{keV}$ is dominated by the $\beta$-decay of ${ }^{113} \mathrm{Cd}$ (half-life $8 \times 10^{15}$ years). At low depths well-understood detector distortions and reconstruction artifacts can be seen.

\section{Summary and Outlook}

Currently COBRA is operating a test setup at the LNGS underground laboratory consisting of $321 \mathrm{~cm}^{3} \mathrm{CdZnTe}$ crystals. In total 64 detectors will be installed and operated by the end of 2013. Meanwhile, considerable progress has been made in understanding detector characteristics and in the improvement of the entire read-out chain. At present a background rate in the region of interest $(2814 \mathrm{keV})$ on the order of 1 counts $/ \mathrm{keV} / \mathrm{kg} / \mathrm{y}$ can be achieved with an energy resolution of $1.5 \%$ (FWHM). The dominant background component has been identified as coming from detector surface contamination. By selecting events by reconstructed interaction depth and pulse shape properties more than $90 \%$ of the background has been removed.

COBRA aims for a large-scale experiment with a total source mass of about $400 \mathrm{~kg}$ of CdZnTe enriched in ${ }^{116} \mathrm{Cd}$ to about $90 \%$. It is likely such an experiment will use larger crystals $\left(2 \times 2 \times 1.5 \mathrm{~cm}^{3}\right)$ which we have begun investigating.
The design goal is to reach a sensitivity to an effective Majorana neutrino mass of less than $50 \mathrm{meV} / \mathrm{c}^{2}$. Depending on the estimate used for the nuclear matrix element [8] this corresponds to a minimum ${ }^{116} \mathrm{Cd}$ half-life sensitivity of $1.0 \times 10^{26}$ to $3.5 \times 10^{26}$ years. To reach this sensitivity, the COBRA experiment needs to reduce the background rate to less than $10^{-3}$ counts $/ \mathrm{keV} / \mathrm{kg} / \mathrm{y}$ while having an energy resolution better than 1.5\% (FWHM). Figure 7 shows sensitivity estimates for a large-scale experiment using 11,000 detectors each with a volume of $6 \mathrm{~cm}^{3}$. The simulation assumes $60 \%$ signal detection efficiency.

The much lower background level required to achieve the conditions depicted in Figure 7 is a significant challenge, similar to that faced by other $0 \nu \beta \beta$-experiments [9-11]. Several methods are currently under investigation to achieve this. The background from lateral surface events could be reduced by modifications in handling procedures, design, and operation of the detectors. Reducing exposure to radon during testing, handling, and storage could reduce the surface contamination by radon daughters (particularly the longlived $\left.{ }^{210} \mathrm{~Pb}\right)$. The use of larger detectors $\left(2 \times 2 \times 1.5 \mathrm{~cm}^{3}\right)$ would reduce this background by increasing the volume to surface area ratio. New designs and operational strategies for the anode grid are also being explored to improve the efficiency of identifying lateral surface events through pulse shape analysis.

Another approach for reducing background is to capitalize on the high granularity of the experiment using coincidence analysis and, thus, restricting events to only occur in single detector units. The presence of the newly installed pulser allows for high-precision coincidence studies. Furthermore, the record of the whole signal pulse shape allows the classification of events by multiple and single interactions within one detector. These techniques are currently under development and not represented in the results presented in this paper. 


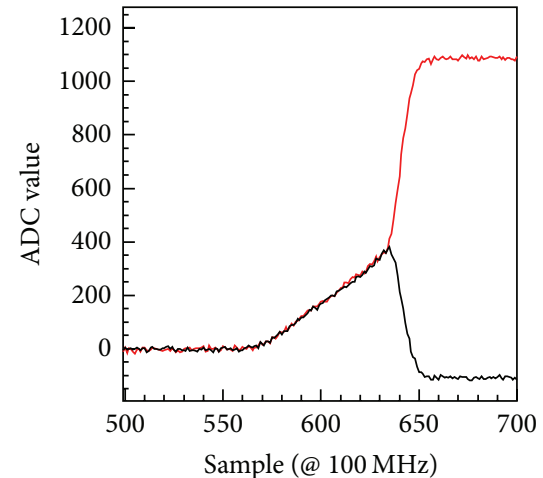

(a)

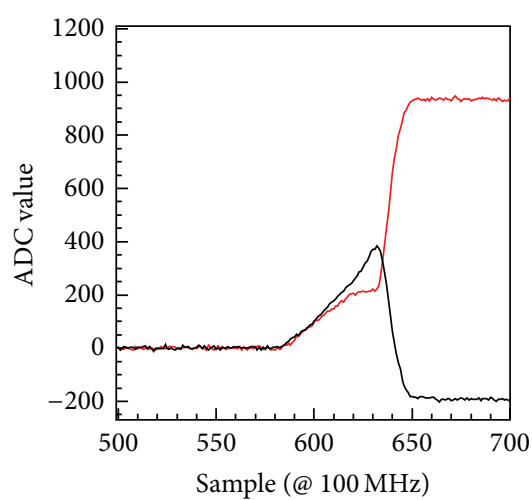

(b)

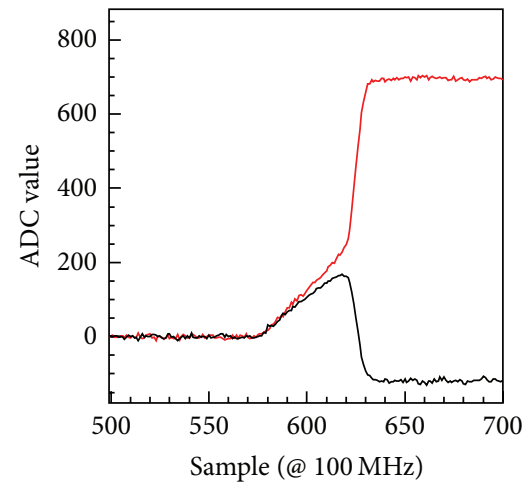

(c)

Figure 5: Pulses from the two anodes. (a) Typical central event. (b) and (c) Lateral surface events showing distortions.

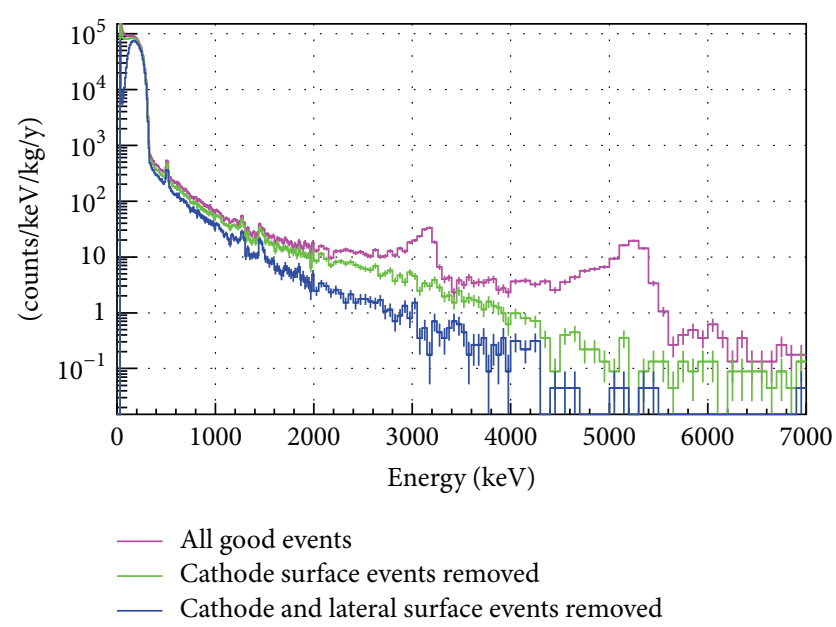

FIGURE 6: Background rate spectrum before and after removal of surface events.

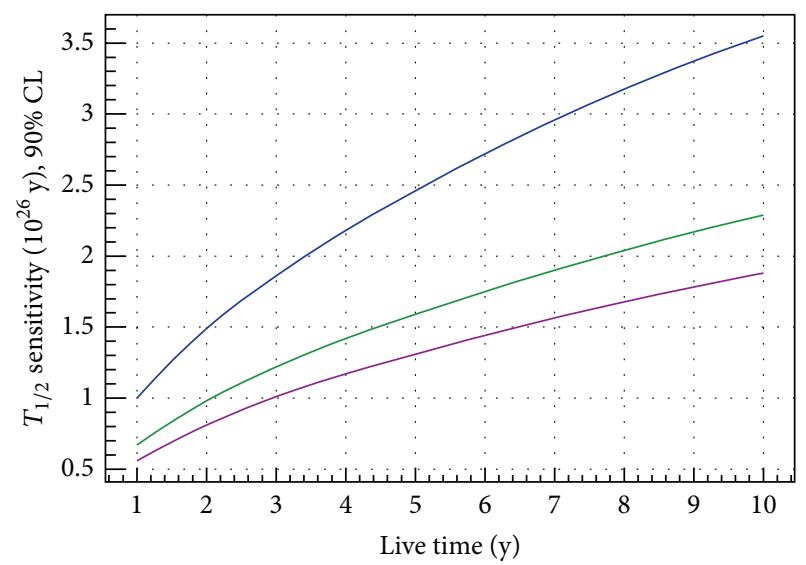

- Background rate $=0.2 \times 10^{-3}$ counts $/ \mathrm{keV} / \mathrm{kg} / \mathrm{y}, \mathrm{FWHM}=1.0 \%$

Background rate $=0.5 \times 10^{-3}$ counts $/ \mathrm{keV} / \mathrm{kg} / \mathrm{y}, \mathrm{FWHM}=1.0 \%$

- Background rate $=0.5 \times 10^{-3}$ counts $/ \mathrm{keV} / \mathrm{kg} / \mathrm{y}, \mathrm{FWHM}=1.5 \%$

FIgURE 7: Sensitivity projections for ${ }^{116} \mathrm{Cd}$ for a large-scale experiment as described in the text, using various background rates and energy resolutions.
The collaboration is also investigating the idea to use liquid scintillators as an ultralow-background environment for $\mathrm{CdZnTe}$ detectors acting as an active veto and as part of coincidence measurements. Other low-background experiments have already shown that the production of liquid scintillators with high radiopurity is possible $[12,13]$.

\section{References}

[1] W. Rodejohann, "Neutrino-less double beta decay and particle physics," International Journal of Modern Physics E, vol. 20, no. 9, pp. 1833-1930, 2011.

[2] K. Zuber, "COBRA-double beta decay searches using CdTe detectors," Physics Letters B, vol. 519, no. 1-2, pp. 1-7, 2001.

[3] O. Schulz, Exploration of new data acquisition and background reduction techniques for the COBRA experiment [doctoral dissertation], Technische Universität Dortmund, 2011, http://hdl.handle.net/2003/29108.

[4] J. V. Dawson, C. Montag, C. Reeve, J. R. Wilson, and K. Zuber, "An investigation on cooling of CZT co-planar grid detectors," Nuclear Instruments and Methods in Physics Research A, vol. 599, no. 2-3, pp. 209-214, 2009.

[5] T. Michel, T. Gleixner, J. Durst, G. Anton, and M. Filipenko, "The potential of CdTe hybrid pixel detectors in the search for the neutrinoless double beta decay of ${ }^{116} \mathrm{Cd}$," This issue of Advances in High Energy Physics.

[6] P. N. Luke, "Single-polarity charge sensing in ionization detectors using coplanar electrodes," Applied Physics Letters, vol. 65, no. 22, pp. 2884-2886, 1994.

[7] M. Fritts, J. Durst, T. Göpfert, T. Wester, and K. Zuber, "Analytical model for event reconstruction in coplanar grid CdZnTe detectors," Nuclear Instruments and Methods in Physics A, vol. 708, pp. 1-6, 2013.

[8] A. Dueck, W. Rodejohann, and K. Zuber, "Neutrinoless double beta decay, the inverted hierarchy, and precision determination of $\theta_{12}$," Physical Review D, vol. 83, no. 11, Article ID 113010, 2011.

[9] The GERDA collaboration, M. Agostini, M. Allardt et al., "The background in the neutrinoless double beta decay experiment GERDA," http://arxiv.org/abs/1306.5084.

[10] M. Auger, D. J. Auty, P. S. Barbeau et al., "Search for neutrinoless double-beta decay in ${ }^{136}$ Xe with EXO-200," Physical Review Letters, vol. 109, no. 3, Article ID 032505, 2012. 
[11] M. Pedretti, M. Barucci, L. Risegari et al., "CUORE Experiment: The search for neutrinoless double beta decay," International Journal of Modern Physics A, vol. 23, no. 21, p. 3395, 2008.

[12] C. Arpesella, H. O. Back, M. Balata et al., "Measurements of extremely low radioactivity levels in BOREXINO," Astroparticle Physics, vol. 18, no. 1, pp. 1-25, 2002.

[13] KamLAND Collaboration, K. Eguchi et al., "First results from KamLAND: evidence for reactor anti-neutrino disappearance," Physical Review Letters, vol. 90, Article ID 021802, 2003. 

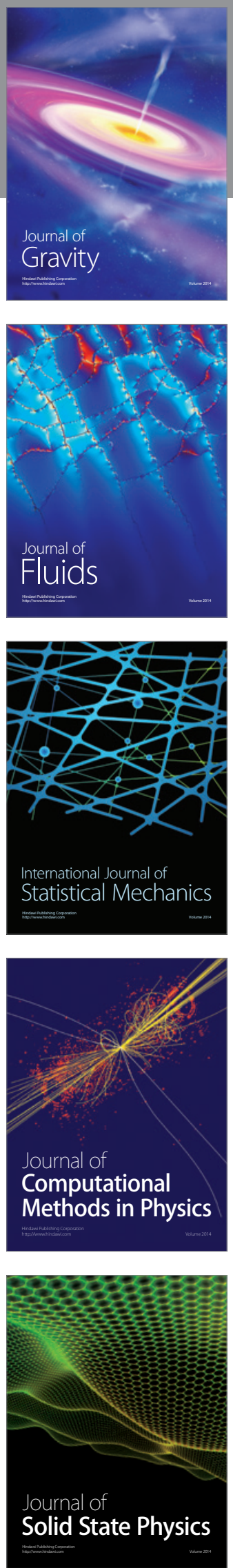

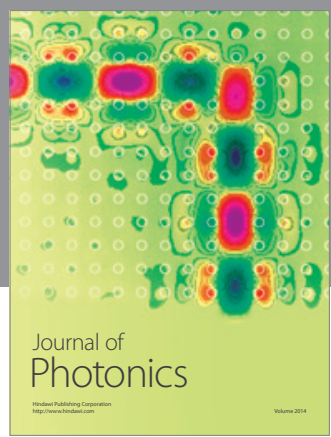

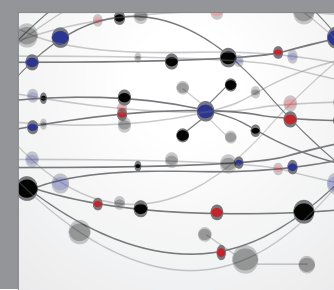

The Scientific World Journal

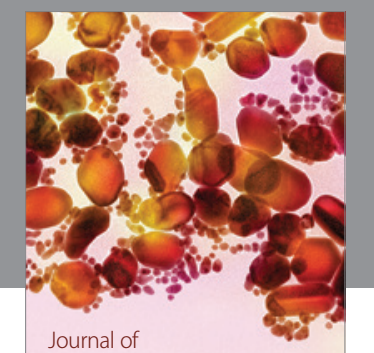

Soft Matter
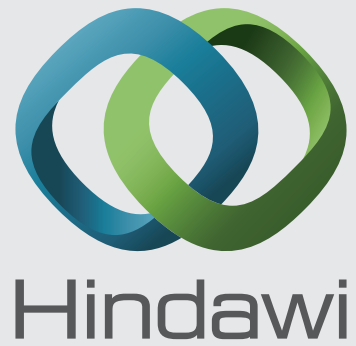

Submit your manuscripts at

http://www.hindawi.com
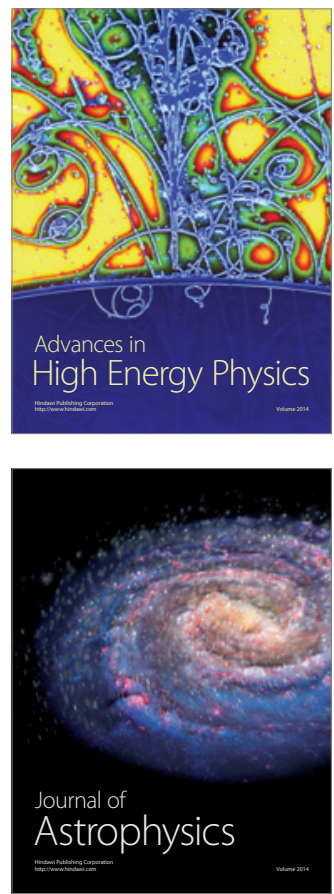
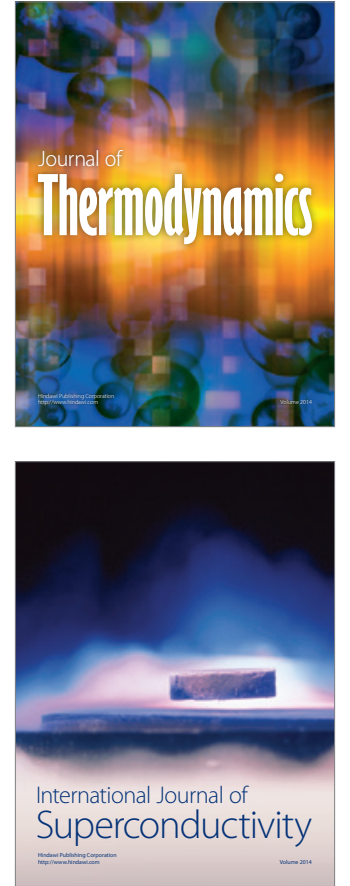
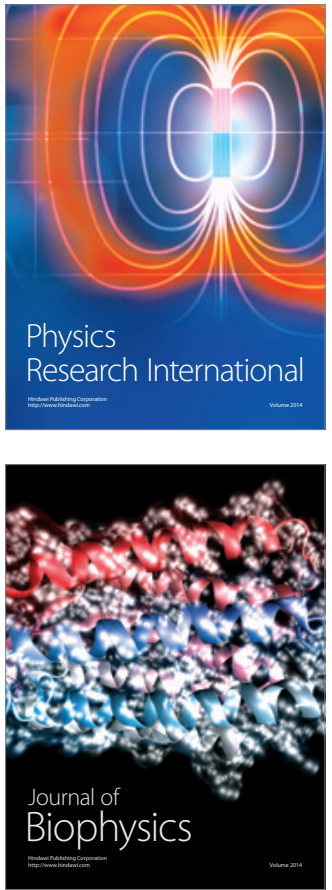
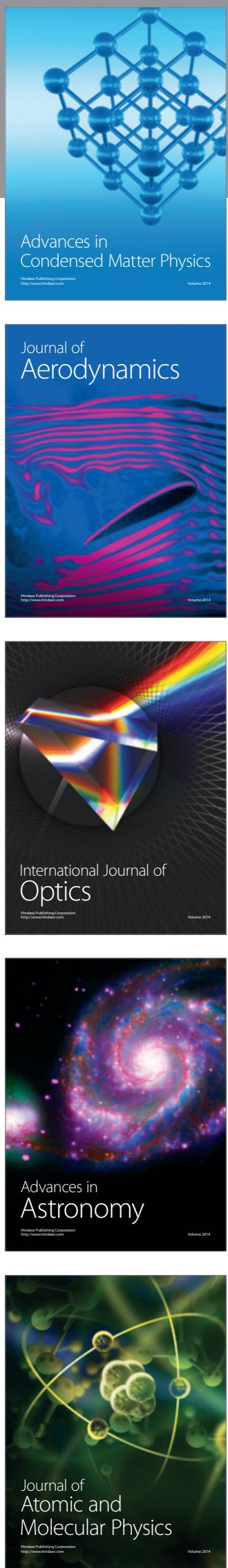